\title{
Computing Coherence Vectors and Correlation Matrices with Application to Quantum Discord Quantification
}

\author{
Jonas Maziero ${ }^{1,2}$ \\ ${ }^{1}$ Departamento de Física, Centro de Ciências Naturais e Exatas, Universidade Federal de Santa Maria, \\ Avenida Roraima 1000, 97105-900 Santa Maria, RS, Brazil \\ ${ }^{2}$ Instituto de Física, Facultad de Ingeniería, Universidad de la República, J. Herrera y Reissig 565, 11300 Montevideo, Uruguay
}

Correspondence should be addressed to Jonas Maziero; jonas.maziero@ufsm.br

Received 17 March 2016; Accepted 14 June 2016

Academic Editor: Kamil Brádler

Copyright (C) 2016 Jonas Maziero. This is an open access article distributed under the Creative Commons Attribution License, which permits unrestricted use, distribution, and reproduction in any medium, provided the original work is properly cited.

Coherence vectors and correlation matrices are important functions frequently used in physics. The numerical calculation of these functions directly from their definitions, which involves Kronecker products and matrix multiplications, may seem to be a reasonable option. Notwithstanding, as we demonstrate in this paper, some algebraic manipulations before programming can reduce considerably their computational complexity. Besides, we provide Fortran code to generate generalized Gell-Mann matrices and to compute the optimized and unoptimized versions of associated Bloch's vectors and correlation matrix in the case of bipartite quantum systems. As a code test and application example, we consider the calculation of Hilbert-Schmidt quantum discords.

\section{Introduction}

Correlation functions are fundamental objects for statistical analysis and are thus ubiquitous in most kinds of scientific inquiries and their applications $[1,2]$. In physics, correlation functions have an important role for research in areas such as quantum optics and open systems [3, 4], phase transitions and condensed matter physics $[5,6]$, and quantum field theory and nuclear and particle physics [7]. Another area in which correlation functions are omnipresent is quantum information science (QIS), an interdisciplinary field that extends the applicability of the classical theories of information, computation, and computational complexity [8-10].

Investigations about the quantum correlations in physical systems have been one of the main catalyzers for developments in QIS [11-15]. There are several guises of quantum correlations, and quantum discord stands among the most promising quantum resources for fueling the quantum advantage [16-26]. When computing or witnessing quantum discord, or other kinds of correlation or quantumness quantifiers, we are frequently faced with the need for calculating coherence vectors and correlation matrices [27-41]. And it is the main aim of this paper to provide formulas for these functions which are amenable for more efficient numerical calculations when compared with the direct implementation of their definitions.

In order to define coherence vectors and correlation matrices, let us consider a composite bipartite system with Hilbert space $\mathscr{H}_{a b}=\mathscr{H}_{a} \otimes \mathscr{H}_{b}$. Hereafter, the corresponding dimensions are denoted by $d_{s}=\operatorname{dim} \mathscr{H}_{s}$ for $s=a b, a, b$. In addition, let $\Gamma_{j}^{s}$, with

$$
\begin{gathered}
\operatorname{Tr}\left(\Gamma_{j}^{s}\right)=0, \\
\operatorname{Tr}\left(\Gamma_{j}^{s} \Gamma_{k}^{s}\right)=2 \delta_{j k}
\end{gathered}
$$

being a basis for the special unitary group $\mathrm{SU}\left(d_{s}\right)$. Any density operator describing the state of the system $\mathscr{H}_{a b}$ can be written in the local basis $\Gamma_{j}^{a} \otimes \Gamma_{k}^{b}$ as follows:

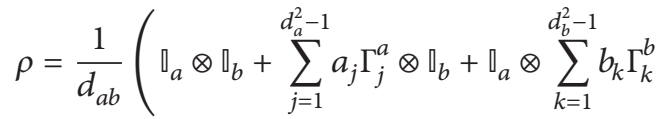

$$
\begin{aligned}
& \left.+\sum_{j=1}^{d_{a}^{2}-1} \sum_{k=1}^{d_{b}^{2}-1} c_{j, k} \Gamma_{j}^{a} \otimes \Gamma_{k}^{b}\right)
\end{aligned}
$$


where

$$
\begin{aligned}
& j=1, \ldots, d_{a}^{2}-1, \\
& k=1, \ldots, d_{b}^{2}-1
\end{aligned}
$$

and $\mathbb{\square}_{s}$ is the identity operator in $\mathscr{H}_{s}$. One can readily verify that the components of the coherence (or Bloch's) vectors $\mathbf{a}=$ $\left(a_{1}, \ldots, a_{d_{a}^{2}-1}\right)$ and $\mathbf{b}=\left(b_{1}, \ldots, b_{d_{b}^{2}-1}\right)$ and of the correlation matrix $C=\left(c_{j, k}\right)$ are given by

$$
\begin{aligned}
a_{j} & =2^{-1} d_{a} \operatorname{Tr}\left(\Gamma_{j}^{a} \otimes \mathbb{I}_{b} \rho\right), \\
b_{k} & =2^{-1} d_{b} \operatorname{Tr}\left(\mathbb{\square}_{a} \otimes \Gamma_{k}^{b} \rho\right), \\
c_{j, k} & =2^{-2} d_{a b} \operatorname{Tr}\left(\Gamma_{j}^{a} \otimes \Gamma_{k}^{b} \rho\right) .
\end{aligned}
$$

It is worth mentioning that the mean value of any observable in $\mathscr{H}_{s}$, for $s=a, b, a b$, can be obtained using these quantities.

In https://github.com/jonasmaziero/LibForQ.git, we provide Fortran code to compute the coherence vectors, correlation matrices, and quantum discord quantifiers we deal with here. Besides these functions, there are other tools therein that may be of interest to the reader. The instructions on how to use the software are provided in the readme file. Related to the content of this section, the subroutine bloch vector_gellmann_unopt $\left(d_{s}, \rho_{s}, \mathbf{s}\right)$ returns the coherence vectors $\mathbf{a}$ or $\mathbf{b}$ and the subroutine corrmat. gellmann_unopt $\left(d_{a}, d_{b}, \rho, C\right)$ computes the correlation matrix $C$. Now, let us notice that if calculated directly from the equations above, for $d_{a}, d_{b} \gg 1$, the computational complexity (CC) to obtain the coherence vectors $\mathbf{a}$ and $\mathbf{b}$ or the correlation matrix $C$ is

$$
\operatorname{CC}(\mathbf{a})=\operatorname{CC}(\mathbf{b})=\operatorname{CC}(C) \approx \mathcal{O}\left(d_{a}^{6} d_{b}^{6}\right) .
$$

The remainder of this paper is structured as follows. In Section 2, we obtain formulas for $\mathbf{a}, \mathbf{b}$, and $C$ which are amenable for more efficient numerical computations. In Section 3 , we test these formulas by applying them in the calculation of Hilbert-Schmidt quantum discords. In Section 4, we make some final remarks about the usefulness and possible applications of the results reported here.

\section{Computing Coherence Vectors and Correlation Matrices}

The partial trace function [42] can be used in order to obtain the reduced states $\rho_{a}=\operatorname{Tr}_{b}(\rho)$ and $\rho_{b}=\operatorname{Tr}_{a}(\rho)$ and to write the components of Bloch's vectors in the form

$$
\begin{aligned}
& a_{j}=2^{-1} d_{a} \operatorname{Tr}\left(\Gamma_{j}^{a} \rho_{a}\right), \\
& b_{k}=2^{-1} d_{b} \operatorname{Tr}\left(\Gamma_{k}^{b} \rho_{b}\right) .
\end{aligned}
$$

Thus, when computing the coherence vectors of the parties $a$ and $b$, we shall solve a similar problem; so let us consider it separately. That is to say, we shall regard a generic density operator written as

$$
\rho_{s}=\frac{1}{d_{s}}\left(\mathbb{\square}_{s}+\sum_{j=1}^{d^{2}-1} s_{j} \Gamma_{j}^{s}\right),
$$

where $s_{j}=2^{-1} d_{s} \operatorname{Tr}\left(\rho_{s} \Gamma_{j}^{s}\right)$.

Now, and for the remainder of this paper, we assume that the matrix elements of regarded density operator $\rho$ in the standard computational basis are given. We want to compute Bloch's vector [43]: $\mathbf{s}=\left(s_{1}, \ldots, s_{d_{s}^{2}-1}\right)$. For the sake of doing that, a particular basis $\Gamma_{j}^{s}$ must be chosen. Here we pick the generalized Gell-Mann matrices, which are presented as follows in three groups [44]:

$$
\begin{gathered}
\Gamma_{j}^{s(1)}=\sqrt{\frac{2}{j(j+1)} \sum_{k=1}^{j+1}(-j)^{\delta_{k, j+1}}|k\rangle\langle k|,} \\
\text { for } j=1, \ldots, d_{s}-1, \\
\Gamma_{(k, l)}^{s(2)}=|k\rangle\langle l|+| l\rangle\langle k|, \quad \text { for } 1 \leq k<l \leq d_{s}, \\
\Gamma_{(k, l)}^{s(3)}=-i(|k\rangle\langle l|-| l\rangle\langle k|), \quad \text { for } 1 \leq k<l \leq d_{s},
\end{gathered}
$$

which are named the diagonal, symmetric, and antisymmetric groups, respectively. The last two groups possess $d_{s}\left(d_{s}-1\right) / 2$ generators each. Any one of these matrices can be obtained by calling the subroutine gellmann $\left(d_{s}, g, k, l\right.$, $\left.\Gamma_{(k, l)}^{s(g)}\right)$. For the first group, $g=1$, we make $j=k$ and, in this case, one can set $l$ to any integer.

It is straightforward seeing that, for the generators above, the corresponding components of Bloch's vector can be expressed directly in terms of the matrix elements of the density operator $\rho_{s}$ as follows:

$$
\begin{gathered}
s_{j}^{(1)}=\frac{d_{s}}{\sqrt{2 j(j+1)}} \sum_{k=1}^{j+1}(-j)^{\delta_{k, j+1}}\left\langle k\left|\rho_{s}\right| k\right\rangle, \\
\quad \text { for } j=1, \ldots, d_{s}-1, \\
s_{(k, l)}^{(2)}=d_{s} \operatorname{Re}\left\langle l\left|\rho_{s}\right| k\right\rangle, \quad \text { for } 1 \leq k<l \leq d_{s}, \\
s_{(k, l)}^{(2)}=d_{s} \operatorname{Im}\left\langle l\left|\rho_{s}\right| k\right\rangle, \quad \text { for } 1 \leq k<l \leq d_{s} .
\end{gathered}
$$

These expressions were implemented in the Fortran subroutine bloch_vector_gellmann $\left(d_{s}, \rho_{s}\right.$, s). With this subroutine and the partial trace function [42], we can compute the coherence vectors $\mathbf{a}$ and $\mathbf{b}$.

We observe that after these simple algebraic manipulations the computational complexity of Bloch's vector turns out to be basically the $\mathrm{CC}$ for the partial trace function. Hence, from [42], we have that, for $d_{a}, d_{b} \gg 1$,

$$
\begin{aligned}
& \mathrm{CC}(\mathbf{a}) \approx \mathcal{O}\left(d_{a}^{2} d_{b}\right), \\
& \mathrm{CC}(\mathbf{b}) \approx \mathcal{O}\left(d_{a} d_{b}^{2}\right) .
\end{aligned}
$$


One detail we should keep in mind when making use of the codes linked to this paper is the convention we apply to the indexes of the components of $\mathbf{s}$. For the first group of generators, $\Gamma_{j}^{s(1)}$, naturally, $j=1, \ldots, d_{s}-1$. We continue with the second group of generators, $\Gamma_{j}^{s(2)}=\Gamma_{(k, l)}^{s(2)}$, by setting $j_{(k, l)=(1,2)}=d_{s}-1+1=d_{s}, j_{(k, l)=(1,3)}=d_{s}+1, \ldots, j_{(k, l)=\left(1, d_{s}\right)}=$ $2\left(d_{s}-1\right), j_{(k, l)=(2,3)}=2\left(d_{s}-1\right)+1, \ldots .$. The same convention is used for the third group of generators, $\Gamma_{j}^{s(3)}=\Gamma_{(k, l)}^{s(3)}$, but here we begin with $j_{(k, l)=(1,2)}=d_{s}-1+2^{-1} d_{s}\left(d_{s}-1\right)+1=d_{s}+$ $2^{-1} d_{s}\left(d_{s}-1\right)$.

Next we address the computation of the correlation matrix $C=\left(c_{j, k}\right)$, which is a $\left(d_{a}^{2}-1\right) \times\left(d_{b}^{2}-1\right)$ matrix that we write in the following form:

$$
C=\left[\begin{array}{lll}
C^{(1,1)} & C^{(1,2)} & C^{(1,3)} \\
C^{(2,1)} & C^{(2,2)} & C^{(2,3)} \\
C^{(3,1)} & C^{(3,2)} & C^{(3,3)}
\end{array}\right],
$$

with the submatrices given as shown below. For convenience, we define the auxiliary variables:

$$
\begin{aligned}
& \iota:=\sqrt{\frac{2}{j(j+1)}}, \\
& \kappa:=\sqrt{\frac{2}{k(k+1)}}, \\
& \varsigma:=\frac{d_{a} d_{b}}{4} .
\end{aligned}
$$

The matrix elements of $C^{(1,1)}$, whose dimension is $\left(d_{a}-\right.$ 1) $\times\left(d_{b}-1\right)$, correspond to the diagonal generators for $a$ and diagonal generators for $b$ :

$$
\begin{aligned}
& c_{j, k}^{(1,1)}=\varsigma \operatorname{Tr}\left(\Gamma_{j}^{a(1)} \otimes \Gamma_{k}^{b(1)} \rho\right)=\varsigma \iota \mathcal{T} \operatorname{Tr}\left(\sum_{m=1}^{j+1}(-j)^{\delta_{m, j+1}}|m\rangle\right. \\
& \cdot\langle m|) \otimes\left(\sum_{p=1}^{k+1}(-k)^{\delta_{p, k+1}}|p\rangle\langle p|\right) \rho \\
& =\varsigma \iota \kappa \sum_{m=1}^{j+1} \sum_{p=1}^{k+1}(-j)^{\delta_{m, j+1}}(-k)^{\delta_{p, k+1}} \\
& \cdot \operatorname{Tr}(|m\rangle\langle m|\otimes| p\rangle\langle p| \rho)=\varsigma \iota \mathcal{L} \sum_{m=1}^{j+1} \sum_{p=1}^{k+1}(-j)^{\delta_{m, j+1}} \\
& \cdot(-k)^{\delta_{p, k+1}}\langle m p|\rho| m p\rangle .
\end{aligned}
$$

The matrix elements of $C^{(1,2)}$, whose dimension is $\left(d_{a}-\right.$ 1) $\times 2^{-1} d_{b}\left(d_{b}-1\right)$, correspond to the diagonal generators for $a$ and symmetric generators for $b$ :

$$
\begin{aligned}
c_{j, k}^{(1,2)} & =\varsigma \operatorname{Tr}\left(\Gamma_{j}^{a(1)} \otimes \Gamma_{k}^{b(2)} \rho\right)=\varsigma \operatorname{Tr}\left(\Gamma_{j}^{a(1)} \otimes \Gamma_{(p, q)}^{b(2)} \rho\right) \\
= & \varsigma \operatorname{Tr}\left(\iota\left(\sum_{m=1}^{j+1}(-j)^{\delta_{m, j+1}}|m\rangle\langle m|\right)\right.
\end{aligned}
$$

$$
\begin{aligned}
& \otimes(|p\rangle\langle q|+| q\rangle\langle p|) \rho)=\varsigma \iota \sum_{m=1}^{j+1}(-j)^{\delta_{m, j+1}} \\
& \cdot(\operatorname{Tr}(|m p\rangle\langle m q| \rho)+\operatorname{Tr}(|m q\rangle\langle m p| \rho)) \\
& =\varsigma \iota \sum_{m=1}^{j+1}(-j)^{\delta_{m, j+1}}\left(\langle m q|\rho| m p\rangle+\langle m q|\rho| m p\rangle^{*}\right) \\
& =2 \varsigma \iota \sum_{m=1}^{j+1}(-j)^{\delta_{m, j+1}} \operatorname{Re}\langle m q|\rho| m p\rangle .
\end{aligned}
$$

The matrix elements of $C^{(1,3)}$, whose dimension is $\left(d_{a}-\right.$ 1) $\times 2^{-1} d_{b}\left(d_{b}-1\right)$, correspond to the diagonal generators for $a$ and antisymmetric generators for $b$ :

$$
\begin{aligned}
& c_{j, k}^{(1,3)}=\varsigma \operatorname{Tr}\left(\Gamma_{j}^{a(1)} \otimes \Gamma_{k}^{b(3)} \rho\right)=\varsigma \operatorname{Tr}\left(\Gamma_{j}^{a(1)} \otimes \Gamma_{(p, q)}^{b(3)} \rho\right) \\
& =-i \varsigma \iota \operatorname{Tr}\left(\left(\sum_{m=1}^{j+1}(-j)^{\delta_{m, j+1}}|m\rangle\langle m|\right)\right. \\
& \otimes(|p\rangle\langle q|-| q\rangle\langle p|) \rho)=-i \varsigma \sum_{m=1}^{j+1}(-j)^{\delta_{m, j+1}} \\
& \cdot(\operatorname{Tr}(|m p\rangle\langle m q| \rho)-\operatorname{Tr}(|m q\rangle\langle m p| \rho)) \\
& =-i \varsigma \iota \sum_{m=1}^{j+1}(-j)^{\delta_{m, j+1}}\left(\langle m q|\rho| m p\rangle-\langle m q|\rho| m p\rangle^{*}\right) \\
& =2 \varsigma \iota \sum_{m=1}^{j+1}(-j)^{\delta_{m, j+1}} \operatorname{Im}\langle m q|\rho| m p\rangle .
\end{aligned}
$$

The matrix elements of $C^{(2,1)}$, whose dimension is $2^{-1} d_{a}\left(d_{a}-1\right) \times\left(d_{b}-1\right)$, correspond to the symmetric generators for $a$ and diagonal generators for $b$ :

$$
\begin{aligned}
& c_{j, k}^{(2,1)}=\varsigma \operatorname{Tr}\left(\Gamma_{j}^{a(2)} \otimes \Gamma_{k}^{b(1)} \rho\right)=\varsigma \operatorname{Tr}\left(\Gamma_{(m, n)}^{a(2)} \otimes \Gamma_{k}^{b(1)} \rho\right) \\
& =\varsigma \operatorname{Tr}((|m\rangle\langle n|+| n\rangle\langle m|) \\
& \left.\otimes \kappa\left(\sum_{p=1}^{k+1}(-k)^{\delta_{p, k+1}}|p\rangle\langle p|\right) \rho\right)=\varsigma \kappa \sum_{p=1}^{k+1}(-k)^{\delta_{p, k+1}} \\
& \cdot(\operatorname{Tr}(|m p\rangle\langle n p| \rho)+\operatorname{Tr}(|n p\rangle\langle m p| \rho)) \\
& =\varsigma \kappa \sum_{p=1}^{k+1}(-k)^{\delta_{p, k+1}}\left(\langle n p|\rho| m p\rangle+\langle n p|\rho| m p\rangle^{*}\right) \\
& =2 \varsigma \kappa \sum_{p=1}^{k+1}(-k)^{\delta_{p, k+1}} \operatorname{Re}\langle n p|\rho| m p\rangle .
\end{aligned}
$$


The matrix elements of $C^{(2,2)}$, whose dimension is $2^{-1} d_{a}\left(d_{a}-1\right) \times 2^{-1} d_{b}\left(d_{b}-1\right)$, correspond to the symmetric generators for $a$ and symmetric generators for $b$ :

$$
\begin{aligned}
& c_{j, k}^{(2,2)}=\varsigma \operatorname{Tr}\left(\Gamma_{j}^{a(2)} \otimes \Gamma_{k}^{b(2)} \rho\right)=\varsigma \operatorname{Tr}\left(\Gamma_{(m, n)}^{a(2)} \otimes \Gamma_{(p, q)}^{b(2)} \rho\right) \\
& =\varsigma \operatorname{Tr}((|m\rangle\langle n|+| n\rangle\langle m|) \otimes(|p\rangle\langle q|+| q\rangle\langle p|) \rho) \\
& =\varsigma(\langle n q|\rho| m p\rangle+\langle m q|\rho| n p\rangle+\langle n p|\rho| m q\rangle \\
& +\langle m p|\rho| n q\rangle)=2 \varsigma(\operatorname{Re}\langle n q|\rho| m p\rangle \\
& +\operatorname{Re}\langle n p|\rho| m q\rangle) .
\end{aligned}
$$

The matrix elements of $C^{(2,3)}$, whose dimension is $2^{-1} d_{a}\left(d_{a}-1\right) \times 2^{-1} d_{b}\left(d_{b}-1\right)$, correspond to the symmetric generators for $a$ and antisymmetric generators for $b$ :

$$
\begin{aligned}
& c_{j, k}^{(2,3)}=\varsigma \operatorname{Tr}\left(\Gamma_{j}^{a(2)} \otimes \Gamma_{k}^{b(3)} \rho\right)=\varsigma \operatorname{Tr}\left(\Gamma_{(m, n)}^{a(2)} \otimes \Gamma_{(p, q)}^{b(3)} \rho\right) \\
& =\varsigma \operatorname{Tr}((|m\rangle\langle n|+| n\rangle\langle m|) \\
& \otimes(-i)(|p\rangle\langle q|-| q\rangle\langle p|) \rho)=-i \varsigma(\langle n q|\rho| m p\rangle \\
& +\langle m q|\rho| n p\rangle-\langle n p|\rho| m q\rangle-\langle m p|\rho| n q\rangle) \\
& =2 \varsigma(\operatorname{Im}\langle n q|\rho| m p\rangle-\operatorname{Im}\langle n p|\rho| m q\rangle) .
\end{aligned}
$$

The matrix elements of $C^{(3,1)}$, whose dimension is $2^{-1} d_{a}\left(d_{a}-1\right) \times\left(d_{b}-1\right)$, correspond to the antisymmetric generators for $a$ and diagonal generators for $b$ :

$$
\begin{aligned}
& c_{j, k}^{(3,1)}=\varsigma \operatorname{Tr}\left(\Gamma_{j}^{a(3)} \otimes \Gamma_{k}^{b(1)} \rho\right)=\operatorname{Tr}\left(\Gamma_{(m, n)}^{a(3)} \otimes \Gamma_{k}^{b(1)} \rho\right) \\
& =\varsigma \operatorname{Tr}(-i(|m\rangle\langle n|-| n\rangle\langle m|) \\
& \left.\otimes \kappa \sum_{p=1}^{k+1}(-k)^{\delta_{p, k+1}}|p\rangle\langle p| \rho\right) \\
& =-i \varsigma \kappa \sum_{p=1}^{k+1}(-k)^{\delta_{p, k+1}}(\langle n p|\rho| m p\rangle-\langle m p|\rho| n p\rangle) \\
& =2 \varsigma \kappa \sum_{p=1}^{k+1}(-k)^{\delta_{p, k+1}} \operatorname{Im}\langle n p|\rho| m p\rangle .
\end{aligned}
$$

The matrix elements of $C^{(3,2)}$, whose dimension is $2^{-1} d_{a}\left(d_{a}-1\right) \times 2^{-1} d_{b}\left(d_{b}-1\right)$, correspond to the antisymmetric generators for $a$ and symmetric generators for $b$ :

$$
\begin{aligned}
c_{j, k}^{(3,2)} & =\varsigma \operatorname{Tr}\left(\Gamma_{j}^{a(3)} \otimes \Gamma_{k}^{b(2)} \rho\right)=\varsigma \operatorname{Tr}\left(\Gamma_{(m, n)}^{a(3)} \otimes \Gamma_{(p, q)}^{b(2)} \rho\right) \\
& =\varsigma \operatorname{Tr}((-i)(|m\rangle\langle n|-| n\rangle\langle m|) \\
& \otimes(|p\rangle\langle q|+| q\rangle\langle p|) \rho)=-i \varsigma(\langle n q|\rho| m p\rangle \\
& -\langle m p|\rho| n q\rangle+\langle n p|\rho| m q\rangle-\langle m q|\rho| n p\rangle) \\
& =2 \varsigma(\operatorname{Im}\langle n q|\rho| m p\rangle+\operatorname{Im}\langle n p|\rho| m q\rangle) .
\end{aligned}
$$

The matrix elements of $C^{(3,3)}$, whose dimension is $2^{-1} d_{a}\left(d_{a}-1\right) \times 2^{-1} d_{b}\left(d_{b}-1\right)$, correspond to antisymmetric generators for $a$ and antisymmetric generators for $b$ :

$$
\begin{aligned}
& c_{j, k}^{(3,3)}=\varsigma \operatorname{Tr}\left(\Gamma_{j}^{a(3)} \otimes \Gamma_{k}^{b(3)} \rho\right)=\varsigma \operatorname{Tr}\left(\Gamma_{(m, n)}^{a(3)} \otimes \Gamma_{(p, q)}^{b(3)} \rho\right) \\
& =\varsigma \operatorname{Tr}(-i(|m\rangle\langle n|-| n\rangle\langle m|) \\
& \otimes(-i)(|p\rangle\langle q|-| q\rangle\langle p|) \rho)=-\varsigma(\langle n q|\rho| m p\rangle \\
& +\langle m p|\rho| n q\rangle-\langle n p|\rho| m q\rangle-\langle m q|\rho| n p\rangle) \\
& =2 \varsigma(\operatorname{Re}\langle n p|\rho| m q\rangle-\operatorname{Re}\langle n q|\rho| m p\rangle) .
\end{aligned}
$$

We remark that when implementing these expressions numerically, for the sake of mapping the local to the global computational basis, we utilize, for example,

$$
|n p\rangle \equiv\left|(n-1) d_{b}+p\right\rangle \text {. }
$$

The subroutine corrmat_gellmann $\left(d_{a}, d_{b}, \rho, C\right)$ returns the correlation matrix $C=\left(c_{j, k}\right)$, as written in (11), associated with the bipartite density operator $\rho$ and computed using the Gell-Mann basis, as described in this section. The convention for the indexes of the matrix elements $c_{j, k}$ is defined in the same way as for the coherence vectors. The computational complexity for $C$, computed via the optimized expressions obtained in this section, is, for $d_{a}, d_{b} \gg 1$,

$$
\mathrm{CC}(C) \approx \mathcal{O}\left(d_{a}^{2} d_{b}^{2}\right) \text {. }
$$

By generating some random density matrices [45], we checked that the expressions and the corresponding code for the unoptimized and optimized versions of $\mathbf{a}, \mathbf{b}$, and $C$ agree. Additional tests shall be presented in the next section, where we calculate some quantum discord quantifiers.

\section{Computing Hilbert-Schmidt Quantum Discords}

The calculation of quantum discord functions (QD) usually involves hard optimization problems $[46,47]$. In the last few years, a large amount of effort has been dedicated towards computing QD analytically, with success being obtained mostly for low-dimensional quantum systems [48-65]. Although not meeting all the required properties for a bona fide QD quantifier [66], the Hilbert-Schmidt discord (HSD) [67],

$$
D_{\mathrm{hs}}^{a}(\rho)=\min _{\rho_{c q}}\left\|\rho-\rho_{c q}\right\|_{2}^{2},
$$

is drawing much attention due to its amenability for analytical computations when compared with most other QD measures. In the last equation, the minimization is performed over the classical-quantum states:

$$
\rho_{c q}=\sum_{j} p_{j}\left|a_{j}\right\rangle\left\langle a_{j}\right| \otimes \rho_{j}^{b},
$$

with $p_{j}$ being a probability distribution, $\left|a_{j}\right\rangle$ being an orthonormal basis for $\mathscr{H}_{a}, \rho_{j}^{b}$ being generic density operators 
defined in $\mathscr{H}_{b}$, and $\|O\|_{2}:=\sqrt{\operatorname{Tr}\left(O^{\dagger} O\right)}$ being the HilbertSchmidt norm of the linear operator $O$, with $\mathrm{O}^{\dagger}$ being the transpose conjugate of $O$.

In this paper, as a basic test for the Fortran code provided to obtain coherence vectors and correlation matrices, we shall compute the following lower bound for the HSD [68]:

$$
D_{\mathrm{hs}}^{a}(\rho)=\sum_{j=d_{a}}^{d_{a}^{2}-1} \lambda_{j}^{a},
$$

where $\lambda_{j}^{a}$ are the eigenvalues, sorted in nonincreasing order, of the $\left(d_{a}^{2}-1\right) \times\left(d_{a}^{2}-1\right)$ matrix:

$$
\Xi_{a}=\frac{2}{d_{a}^{2} d_{b}}\left(\mathbf{a a}^{t}+\frac{2}{d_{b}} C C^{t}\right) .
$$

In the above equation, $t$ stands for the transpose of a vector or matrix. We observe that the other version of the HSD, $D_{\mathrm{hs}}^{b}$, can be obtained from the above equations simply by exchanging $a$ and $b$ and using $C^{t} C$ instead of $C C^{t}$.

It is interesting that, as was proved in [69], a bipartite state $\rho$, with polarization vectors $\mathbf{a}$ and $\mathbf{b}$ and correlation matrix $C$, is classical-quantum if and only if there exists a $\left(d_{a}-1\right)$ dimensional projector $\Pi_{a}$ in the space $\mathbb{R}^{d_{a}^{2}-1}$ such that

$$
\begin{aligned}
\Pi_{a} \mathbf{a} & =\mathbf{a}, \\
\Pi_{a} C & =C .
\end{aligned}
$$

Based on this fact, an ameliorated version for the HilbertSchmidt quantum discord (AHSD) was proposed [69]:

$$
D_{\text {hsa }}^{a}(\rho):=\min _{\Pi_{a}}\left\|\Upsilon_{a}-\Pi_{a} \Upsilon_{a}\right\|_{2}^{2},
$$

with the matrix $\Upsilon_{a}$ defined as

$$
\Upsilon_{a}:=\sqrt{\frac{2}{d_{a}^{2} d_{b}}}\left(f(b) \mathbf{a} g(b) \sqrt{\frac{2}{d_{b}}} C\right)
$$

where $f$ and $g$ are arbitrary functions of $b \equiv\|\mathbf{b}\|_{2}$. Then, by setting $f(b)=g(b)=P\left(\rho_{b}\right)$ and using the purity,

$$
P\left(\rho_{b}\right):=\operatorname{Tr}\left(\rho_{b}^{2}\right) \sum_{j, k}\left|\rho_{j, k}^{b}\right|^{2},
$$

to address the problem of noncontractivity of the HilbertSchmidt distance, the following analytical formula was presented [69]:

$$
D_{\text {hsa }}^{a}(\rho)=\frac{1}{P\left(\rho_{b}\right)} \sum_{j=d_{a}}^{d_{a}^{2}-1} \lambda_{j}^{a}=\frac{D_{\text {hs }}^{a}(\rho)}{P\left(\rho_{b}\right)} .
$$

Thus both discord quantifiers $D_{\mathrm{hs}}^{a}$ and $D_{\mathrm{hsa}}^{a}$ are, at the end of the day, obtained from the eigenvalues $\lambda_{j}^{a}$. And the computation of these eigenvalues requires the knowledge of the coherence vector $\mathbf{a}$ (or $\mathbf{b}$ ) and of the correlation matrix $C$. These QD measures were implemented in

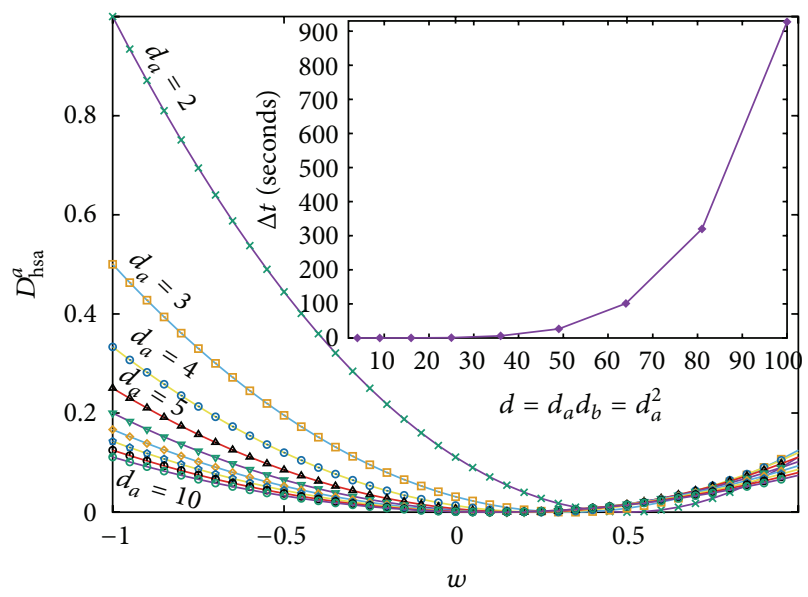

FIgURE 1: The points are the values of the ameliorated HilbertSchmidt quantum discord of Werner states computed numerically using (32). The lines are the corresponding values of the AHSD plotted via the analytical formula: $D_{\mathrm{hsa}}^{a}\left(\rho^{w}\right)=d_{a} D_{\mathrm{hs}}^{a}\left(\rho^{w}\right)=\left(d_{a} w-\right.$ $1)^{2} /\left(\left(d_{a}-1\right)\left(d_{a}+1\right)^{2}\right)$. Due to the symmetry of $\rho^{w}$, here $D_{\text {hsa }}^{a}\left(\rho^{w}\right)=$ $D_{\text {hsa }}^{b}\left(\rho^{w}\right)$. In the inset the difference between the times taken by the two methods to compute the AHSD for a fixed value of $d_{a}$ is shown. We see clearly here that our optimized algorithm gives an exponential speedup against the brute force calculation of Bloch's vectors and correlation matrix.

the Fortran functions discord_hs (ssys, $d_{a}, d_{b}, \rho$ ) and discord_hsa (ssys, $d_{a}, d_{b}, \rho$ ), where ssys $=$ "s", with $s=a, b$, specifies which version of the quantum discord is to be computed. As an example, let us use the formulas provided in this paper and the associated code to compute the HSD and AHSD of Werner states in $\mathscr{H}_{a} \otimes \mathscr{H}_{b}\left(\right.$ with $\left.d_{a}=d_{b}\right)$ :

$$
\rho^{w}=\frac{d_{a}-w}{d_{a}\left(d_{a}^{2}-1\right)} \mathbb{q}_{a} \otimes \mathbb{\square}_{b}+\frac{d_{a} w-1}{d_{a}\left(d_{a}^{2}-1\right)} F,
$$

where $w \in[-1,1]$ and $F=\sum_{j, k=1}^{d_{a}}|j k\rangle\langle k j|$. The reduced states of $\rho^{w}$ are $\mathbb{1}_{s} / d_{s}$, whose purity is $P\left(\rho_{s}\right)=1 / d_{s}$. The results for the HSD and AHSD of $\rho^{w}$ are presented in Figure 1.

\section{Concluding Remarks}

In this paper, we addressed the problem of computing coherence vectors and correlations matrices. We obtained formulas for these functions which make possible considerably more efficient numerical implementation when compared with the direct use of their definitions. We provided Fortran code to calculate all the quantities regarded in this paper. As a test for our formulas and code, we computed Hilbert-Schmidt quantum discords of Werner states. It is important to observe that although our focus here was in quantum information science, the tools provided can find application in other areas, such as the calculation of order parameters and correlations functions for the study of phase transitions in discrete quantum or classical systems. 


\section{Competing Interests}

The author declares that there are no competing interests regarding the publication of this paper.

\section{Acknowledgments}

This work was supported by the following Brazilian funding agencies: Conselho Nacional de Desenvolvimento Científico e Tecnológico (CNPq) (Processes 441875/2014-9 and 303496/2014-2), Instituto Nacional de Ciência e Tecnologia de Informação Quântica (INCT-IQ) (Process 2008/57856-6), and Coordenação de Desenvolvimento de Pessoal de Nível Superior (CAPES) (Process 6531/2014-08). The author gratefully acknowledges the hospitality of the Physics Institute and Laser Spectroscopy Group at Universidad de la República, Uruguay.

\section{References}

[1] E. T. Jaynes, Probability Theory, the Logic of Science, Cambridge University Press, New York, NY, USA, 2003.

[2] M. A. Carlton and J. L. Devore, Probability with Applications in Engineering, Science, and Technology, Springer Texts in Statistics, Springer, New York, NY, USA, 2014.

[3] C. Gerry and P. Knight, Introductory Quantum Optics, Cambridge University Press, New York, NY, USA, 2005.

[4] H.-P. Breuer and F. Petruccione, The Theory of Open Quantum Systems, Oxford University Press, New York, NY, USA, 2002.

[5] S. Sachdev, Quantum Phase Transitions, Cambridge University Press, New York, NY, USA, 2011.

[6] J. Hutchinson, J. P. Keating, and F. Mezzadri, "On relations between one-dimensional quantum and two-dimensional classical spin systems," Advances in Mathematical Physics, vol. 2015, Article ID 652026, 18 pages, 2015.

[7] L. Álvarez-Gaumé and M. Vázquez-Mozo, An Invitation to Quantum Field Theory, Springer, Berlin, Germany, 2012.

[8] M. A. Nielsen and I. L. Chuang, Quantum Computation and Quantum Information, Cambridge University Press, Cambridge, UK, 2000.

[9] J. Preskill, Quantum Information and Computation, http://theory.caltech.edu/people/preskill/ph229/.

[10] M. M. Wilde, Quantum Information Theory, Cambridge University Press, Cambridge, UK, 2013.

[11] M. D. Reid, P. D. Drummond, W. P. Bowen et al., "The Einstein-Podolsky-Rosen paradox: from concepts to applications," Reviews of Modern Physics, vol. 81, no. 4, pp. 1727-1751, 2009.

[12] R. Horodecki, P. Horodecki, and M. H. Horodecki, "Quantum entanglement," Reviews of Modern Physics, vol. 81, no. 2, pp. 865-942, 2009.

[13] M. Li, S.-M. Fei, and X. Li-Jost, "Quantum entanglement: separability, measure, fidelity of teleportation, and distillation," Advances in Mathematical Physics, vol. 2010, Article ID 301072, 57 pages, 2010.

[14] L. C. Céleri, J. Maziero, and R. M. Serra, "Theoretical and experimental aspects of quantum discord and related measures," International Journal of Quantum Information, vol. 9, no. 7-8, pp. 1837-1873, 2011.

[15] K. Modi, A. Brodutch, H. Cable, T. Paterek, and V. Vedral, "The classical-quantum boundary for correlations: discord and related measures," Reviews of Modern Physics, vol. 84, no. 4, article 1655, 2012.

[16] D. O. Soares-Pinto, R. Auccaise, J. Maziero, A. Gavini-Viana, R. M. Serra, and L. C. Céleri, "On the quantumness of correlations in nuclear magnetic resonance," Philosophical Transactions of the Royal Society A: Mathematical, Physical and Engineering Sciences, vol. 370, no. 1976, pp. 4821-4836, 2012.

[17] M. Ávila, G. H. Sun, and A. L. Salas-Brito, "Scales of time where the quantum discord allows an efficient execution of the DQC1 algorithm," Advances in Mathematical Physics, vol. 2014, Article ID 367905, 4 pages, 2014.

[18] A. Streltsov, Quantum Correlations Beyond Entanglement, and Their Role in Quantum Information Theory, Springer Briefs in Physics, 2015.

[19] A. Datta, A. Shaji, and C. M. Caves, "Quantum discord and the power of one qubit," Physical Review Letters, vol. 100, no. 5, Article ID 050502, 2008.

[20] D. Cavalcanti, L. Aolita, S. Boixo, K. Modi, M. Piani, and A. Winter, "Operational interpretations of quantum discord," Physical Review A, vol. 83, Article ID 032324, 2011.

[21] S. Pirandola, "Quantum discord as a resource for quantum cryptography," Scientific Reports, vol. 4, article 6956, 2014.

[22] L. Roa, J. C. Retamal, and M. Alid-Vaccarezza, "Dissonance is required for assisted optimal state discrimination," Physical Review Letters, vol. 107, no. 8, Article ID 080401, 2011.

[23] M. Piani, V. Narasimhachar, and J. Calsamiglia, "Quantumness of correlations, quantumness of ensembles and quantum data hiding," New Journal of Physics, vol. 16, Article ID 113001, 2014.

[24] B. Dakić, Y. O. Lipp, X. Ma et al., "Quantum discord as resource for remote state preparation," Nature Physics, vol. 8, no. 9, pp. 666-670, 2012.

[25] D. Girolami, A. M. Souza, V. Giovannetti et al., "Quantum discord determines the interferometric power of quantum states," Physical Review Letters, vol. 112, no. 21, Article ID 210401, 2014.

[26] T. K. Chuan, J. Maillard, K. Modi, T. Paterek, M. Paternostro, and M. Piani, "Quantum discord bounds the amount of distributed entanglement," Physical Review Letters, vol. 109, no. 7, Article ID 070501, 2012.

[27] J. Maziero and R. M. Serra, "Classicality witness for two-qubit states," International Journal of Quantum Information, vol. 10, no. 3, Article ID 1250028, 8 pages, 2012.

[28] R. Auccaise, J. Maziero, L. C. Céleri et al., "Experimentally witnessing the quantumness of correlations," Physical Review Letters, vol. 107, no. 7, Article ID 070501, 2011.

[29] F. M. Paula, I. A. Silva, J. D. Montealegre et al., "Observation of environment-induced double sudden transitions in geometric quantum correlations," Physical Review Letters, vol. 111, no. 25, Article ID 250401, 2013.

[30] I. A. Silva, D. Girolami, R. Auccaise et al., "Measuring bipartite quantum correlations of an unknown state," Physical Review Letters, vol. 110, no. 14, Article ID 140501, 2013.

[31] G. H. Aguilar, O. Jiménez Farías, J. Maziero, R. M. Serra, P. H. Souto Ribeiro, and S. P. Walborn, "Experimental estimate of a classicality witness via a single measurement," Physical Review Letters, vol. 108, no. 6, Article ID 063601, 2012.

[32] A. Bayat and S. Bose, "Entanglement transfer through an antiferromagnetic spin chain," Advances in Mathematical Physics, vol. 2010, Article ID 127182, 11 pages, 2010.

[33] M. Cianciaruso, T. R. Bromley, W. Roga, R. Lo Franco, and G. Adesso, "Universal freezing of quantum correlations within the 
geometric approach," Scientific Reports, vol. 5, Article ID 10177, 2015.

[34] T. R. Bromley, M. Cianciaruso, R. Lo Franco, and G. Adesso, "Unifying approach to the quantification of bipartite correlations by Bures distance," Journal of Physics A: Mathematical and Theoretical, vol. 47, no. 40, Article ID 405302, 20 pages, 2014.

[35] B. Aaronson, R. L. Franco, G. Compagno, and G. Adesso, "Hierarchy and dynamics of trace distance correlations," New Journal of Physics, vol. 15, Article ID 093022, 2013.

[36] B. Aaronson, R. Lo Franco, and G. Adesso, "Comparative investigation of the freezing phenomena for quantum correlations under nondissipative decoherence," Physical Review A, vol. 88, no. 1, Article ID 012120, 2013.

[37] B. Bellomo, G. L. Giorgi, F. Galve, R. Lo Franco, G. Compagno, and R. Zambrini, "Unified view of correlations using the squarenorm distance," Physical Review A-Atomic, Molecular, and Optical Physics, vol. 85, no. 3, Article ID 032104, 2012.

[38] B. Bellomo, R. Lo Franco, and G. Compagno, "Dynamics of geometric and entropic quantifiers of correlations in open quantum systems," Physical Review A, vol. 86, no. 1, Article ID 012312, 2012.

[39] R. Lo Franco, B. Bellomo, E. Andersson, and G. Compagno, "Revival of quantum correlations without system-environment back-action," Physical Review A, vol. 85, no. 3, Article ID 032318, 2012.

[40] J.-S. Xu, K. Sun, C.-F. Li et al., "Experimental recovery of quantum correlations in absence of system-environment backaction," Nature Communications, vol. 4, article 2851, 2013.

[41] R. L. Franco, B. Bellomo, S. Maniscalco, and G. Compagno, "Dynamics of quantum correlations in two-qubit systems within non-Markovian environments," International Journal of Modern Physics B: Condensed Matter Physics; Statistical Physics; Atomic, Molecular and Optical Physics, vol. 27, no. 1-3, Article ID 1345053, 20 pages, 2013.

[42] J. Maziero, "Computing partial traces and reduced density matrices," http://arxiv.org/abs/1601.07458.

[43] E. Brüning, H. Mäkelä, A. Messina, and F. Petruccione, "Parametrizations of density matrices," Journal of Modern Optics, vol. 59, no. 1, pp. 1-20, 2012.

[44] R. A. Bertlmann and P. Krammer, "Bloch vectors for qudits," Journal of Physics A: Mathematical and Theoretical, vol. 41, no. 23, Article ID 235303, 21 pages, 2008.

[45] J. Maziero, "Fortran code for generating random probability vectors, unitaries, and quantum states," Frontiers in ICT, vol. 3 , article 4, 2016.

[46] Y. Huang, "Computing quantum discord is NP-complete," New Journal of Physics, vol. 16, Article ID 033027, 2014.

[47] T. Chanda, T. Das, D. Sadhukhan, A. K. Pal, A. Sen De, and U. Sen, "Reducing computational complexity of quantum correlations," Physical Review A, vol. 92, no. 6, Article ID 062301, 2015.

[48] S. Luo, "Quantum discord for two-qubit systems," Physical Review A, vol. 77, Article ID 042303, 2008.

[49] S. Luo and Q. Zhang, "Observable correlations in two-qubit states," Journal of Statistical Physics, vol. 136, no. 1, pp. 165-177, 2009.

[50] M. Ali, A. R. P. Rau, and G. Alber, "Quantum discord for twoqubit X states," Physical Review A, vol. 81, Article ID 042105, 2010.

[51] J. Maziero, L. C. Celeri, and R. M. Serra, "Symmetry aspects of quantum discord,” https://arxiv.org/abs/1004.2082.
[52] D. Girolami and G. Adesso, "Quantum discord for general twoqubit states: analytical progress," Physical Review A, vol. 83, no. 5, Article ID 052108, 2011.

[53] Q. Chen, C. Zhang, S. Yu, X. X. Yi, and C. H. Oh, "Quantum discord of two-qubit $X$ state," Physical Review A, vol. 84, no. 4, Article ID 042313, 2011.

[54] A. S. M. Hassan, B. Lari, and P. S. Joag, "Tight lower bound to the geometric measure of quantum discord," Physical Review A, vol. 85, no. 2, Article ID 024302, 2012.

[55] F. Ciccarello, T. Tufarelli, and V. Giovannetti, “Toward computability of trace distance discord," New Journal of Physics, vol. 16, Article ID 013038, 2014.

[56] D. Spehner and M. Orszag, "Geometric quantum discord with Bures distance: the qubit case," Journal of Physics A: Mathematical and Theoretical, vol. 47, no. 3, Article ID 035302, 20 pages, 2014.

[57] M. A. Jafarizadeh, N. Karimi, and H. Zahir, "Quantum discord for generalized bloch sphere states," European Physical Journal $D$, vol. 68, no. 5, article 136, 2014.

[58] M. Daoud, R. Ahl Laamara, and S. Seddik, "Hilbert-Schmidt measure of pairwise quantum discord for three-qubit $X$ states," Reports on Mathematical Physics, vol. 76, no. 2, pp. 207-230, 2015.

[59] A. Beggi, F. Buscemi, and P. Bordone, "Analytical expression of genuine tripartite quantum discord for symmetrical X-states," Quantum Information Processing, vol. 14, no. 2, pp. 573-592, 2015.

[60] Y.-K. Wang, N. Jing, S.-M. Fei, Z.-X. Wang, J.-P. Cao, and H. Fan, "One-way deficit of two-qubit $X$ states," Quantum Information Processing, vol. 14, no. 7, pp. 2487-2497, 2015.

[61] Z. Ma, Z. Chen, F. F. Fanchini, and S.-M. Fei, "Quantum discord for d 2 systems," Scientific Reports, vol. 5, Article ID 10262, 2015.

[62] N. Jing, X. Zhang, and Y.-K. Wang, "Comment on: oneway deficit of two qubit X states," Quantum Information Processing, vol. 14, pp. 4511-4521, 2015.

[63] G. Li, Y. Liu, H. Tang, X. Yin, and Z. Zhang, "Analytic expression of quantum correlations in qutrit Werner states undergoing local and nonlocal unitary operations," Quantum Information Processing, vol. 14, no. 2, pp. 559-572, 2015.

[64] P. C. Obando, F. M. Paula, and M. S. Sarandy, "Trace-distance correlations for X states and the emergence of the pointer basis in Markovian and non-Markovian regimes," Physical Review A, vol. 92, no. 3, Article ID 032307, 2015.

[65] Y. Huang, "Quantum discord for two-qubit X states: analytical formula with very small worst-case error," Physical Review A, vol. 88, no. 1, Article ID 014302, 2013.

[66] A. Brodutch and K. Modi, "Criteria for measures of quantum correlations," Quantum Information \& Computation, vol. 12, no. 9-10, article 0721, 2012.

[67] B. Dakić, V. Vedral, and C. Brukner, "Necessary and sufficient condition for nonzero quantum discord," Physical Review Letters, vol. 105, no. 19, Article ID 190502, 2010.

[68] S. Luo and S. Fu, "Geometric measure of quantum discord," Physical Review A, vol. 82, no. 3, Article ID 034302, 4 pages, 2010.

[69] S. J. Akhtarshenas, H. Mohammadi, S. Karimi, and Z. Azmi, "Computable measure of quantum correlation," Quantum Information Processing, vol. 14, no. 1, pp. 247-267, 2015. 


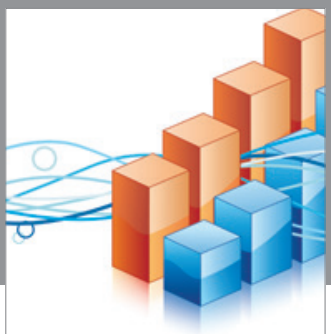

Advances in

Operations Research

vatem alat4

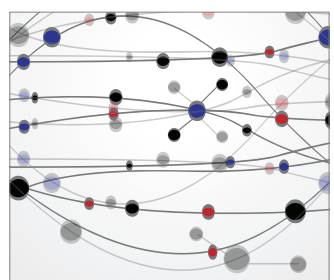

\section{The Scientific} World Journal
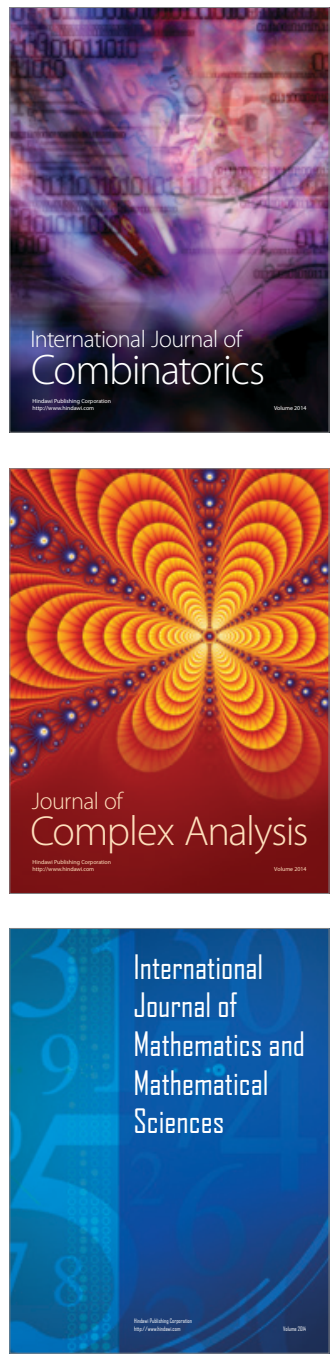
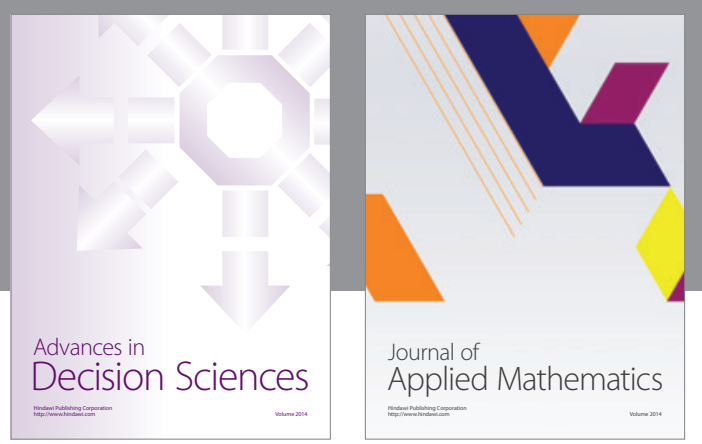

Algebra

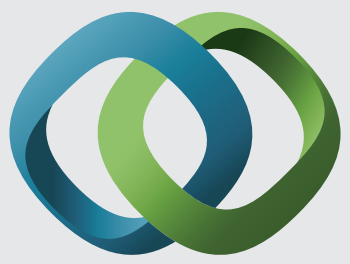

\section{Hindawi}

Submit your manuscripts at

http://www.hindawi.com
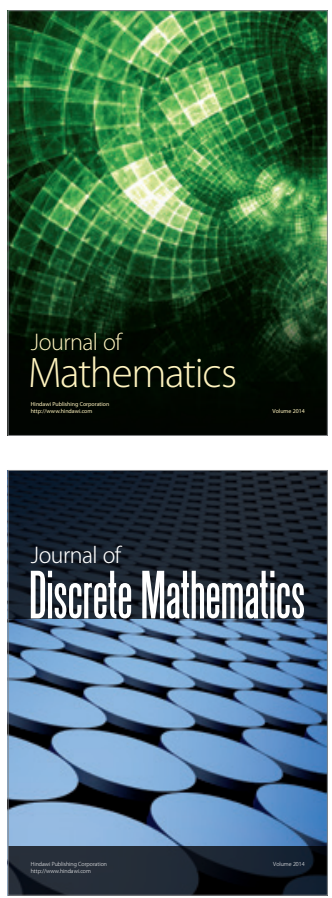

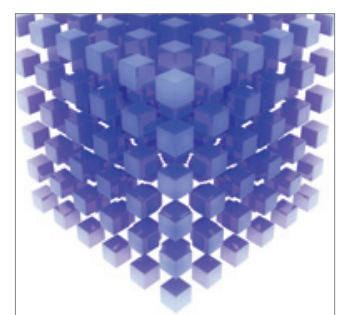

Mathematical Problems in Engineering
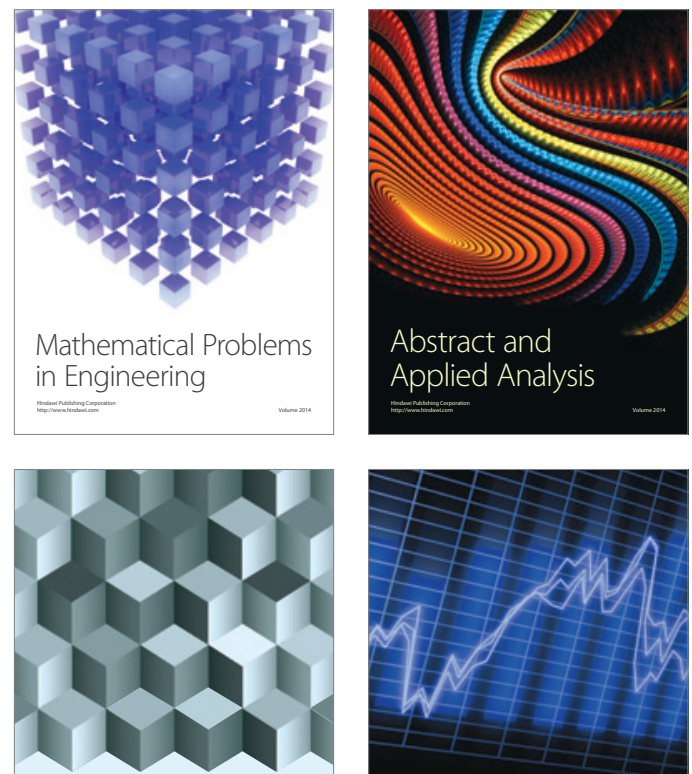

Journal of

Function Spaces

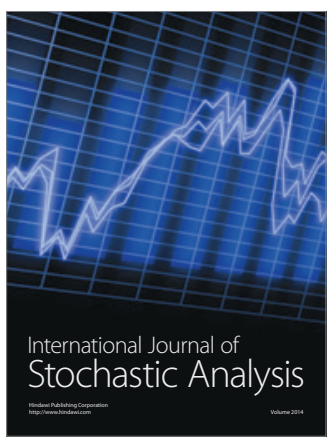

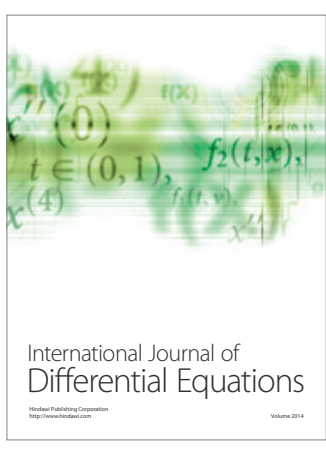
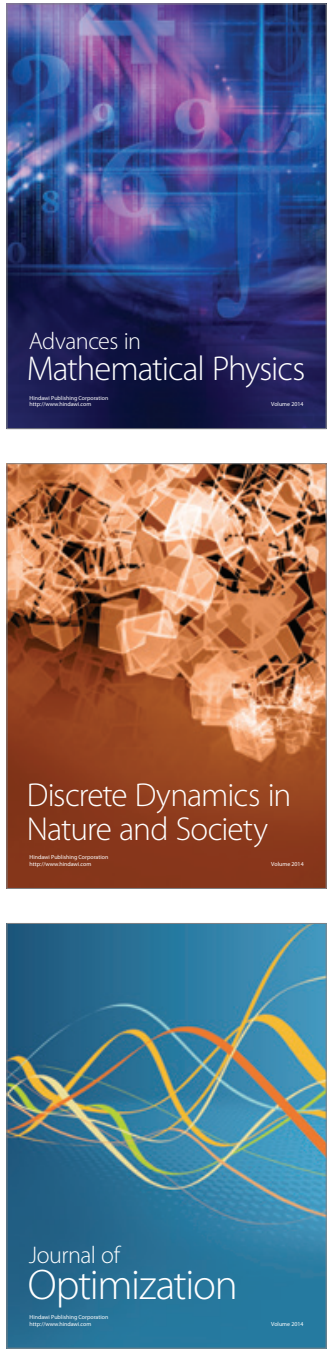